\title{
New Consistent Numerical Modelling of a Travelling Accelerating Concentrated Mass
}

\author{
Bartlomiej Dyniewicz ${ }^{1}$, Czeslaw I. Bajer ${ }^{1,2}$ \\ ${ }^{1}$ Institute of Fundamental Technological Research, Polish Academy of Sciences, Warsaw, Poland \\ ${ }^{2}$ The Faculty of Automotive and Construction Machinery Engineering, Warsaw University of Technology, Warsaw, Poland \\ Email: bdynie@ippt.gov.pl
}

Received September 4, 2012; revised October 6, 2012; accepted October 18, 2012

\begin{abstract}
This paper deals with vibrations of structures subjected to moving inertial loads. In literature structures are usually subjected to massless forces. In numerical applications, however, the direct influence of the inertia of a moving object is usually neglected since the characteristic matrices, although simple, can not be easily derived. The paper presents a direct, non-iterative treatment of the motion of a mass along the finite element edge. The general characteristic matrices of finite elements that carry an inertial particle are given and can be applied directly to almost all types of structures. Numerical tests and a comparison with examples from the literature and especially with analytical results, prove the efficiency and accuracy of our analysis.
\end{abstract}

Keywords: Vibrations; Moving Mass; Moving Inertial Load; Time Integration

\section{Introduction}

Increasing the speed and weight of vehicle gives rise to new problems, poorly studied as of yet. These are mainly the adverse exploitation effects caused by wave phenomena. Problems show up when we perform computer simulations. In the case of wave problems, the numerical description of moving inertial loads requires great mathematical care. Otherwise we get a wrong solution. There is no commercial computing package that would enable the direct simulation of moving loads, both gravity and inertia. Perhaps this is due to the lack of universal matrices describing such moving inertial loads.

Analytical and semi-analytical results for simply supported or cantilever beams under a moving mass are known [1-4]. This solution is based on the classical separation of variables. It results in the finite trigonometric series which fulfils boundary conditions. The interesting solution to the problem of interaction between moving bodies and structures is essentially simplified owing to separation of the two objects [5]. However, with more realistic structures usually the finite element approach is applied. Accurate results are fundamental for decisions at a design stage. An accurate estimation of the dynamic influence is essential for proper modelling. Accurate results are important not only for increasing the durability and reliability of systems: predicting the level of the dynamic response of structures under a moving load allows of protecting the environment, especially populated urban centres or historical places.

The development of computer methods has led to a series of works on numerical calculations, especially using the finite element method. This method is much more versatile than analytical or semi-analytical methods. Papers discussing moving loads with constant or periodic amplitude $[6,7]$ rely on the step by step modification of the right hand side vector of the resulting system of algebraic equations. The results have been presented in papers devoted to modelling the motion of a vehicle as a group of oscillators [8-10]. These problems require the coincidence of displacements and forces of two subsystems: the main structure and the moving oscillator. For the balance of the respective quantities in both systems, a simple iterative procedure is applied. The convergence of such an iterative scheme is limited to a certain range of parameters, such as the travelling velocity, stiffness of the structure, inertia, and especially the time step. Otherwise the iterative procedure must be more complex and time consuming. In addition, the oscillator can not replace the point mass. In the limiting case of infinite spring stiffness, it has been shown that the moving oscillator problem for a simply supported beam is not equivalent, in a strict sense, to the moving mass problem [11].

The insertion of the inertia of the moving load effect requires not only modification of the right hand side vector, but also selected parts of the global inertia, damping, and stiffness matrices of the system, at every 
time step. The first study to discuss the influence of the inertia of a moving mass was reported in [12]. An inertial load moving at a constant speed on the Euler beam was considered. Further works [13-15] are also related to beams or plates in which the nodal parameters are interpolated by cubic polynomials. Let us apply them to the pure hyperbolic equation of the string vibrations. Unfortunately, the results there are wrong. Simulations fail because of the divergence of the solution.

In literature, we can also find examples of the spacetime finite element method applied to moving loads. The idea is based on the equilibrium of the energy of a structure in time interval (Figure 1).

It is based on the weak formulation and allows us to solve much more complex problems, including moving concentrated physical parameters. This approach was successfully applied to simple moving mass problem, solved by discrete methods $[16,17]$.

Although the space-time approach in the case of a differential equation with constant coefficients and stationary discretization results in practically the same algorithms as classical time integration methods, most engineers chose methods of the Newmark group for computations. A simple modification of the inertia matrix by adding the $\mathrm{AD}$ hoc moving mass lumping in nodes in the Newmark algorithm or direct differentiation of the acceleration of the mass particle according to the moving argument and then incorporation of the resulting matrices to the solution scheme fail.

The practice of numerical simulations, however, requires simplicity and efficiency of procedures. Characteristic matrices for an inertial particle should be capable of being easily incorporated into computer procedures. Thus all existing commercial codes would gain new possibilities of calculations. We will focus our attention on this aim.

We will derive correct matrices which can be applied to discrete solutions by methods of the Newmark group of all types of differential equations, especially a string, the Bernoulli-Euler beam or the Timoshenko beam. They differ from matrices used in literature. Numerical tests

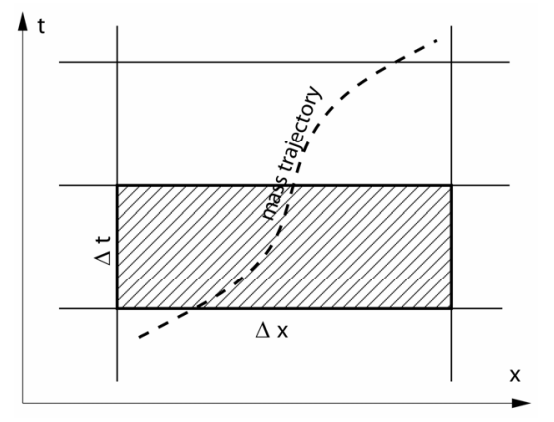

Figure 1. The mass trajectory in space and time. and a comparison with test examples published so far are given.

\section{Mathematical Model}

Let us consider differential equations of structures containing a concentrated mass. We will focus our attention on the term which describes forces induced by a moving inertial particle. In the case of a string we can write the equation in the form:

$$
\begin{aligned}
& -N \frac{\partial^{2} w(x, t)}{\partial x^{2}}+\rho A \frac{\partial^{2} w(x, t)}{\partial t^{2}} \\
= & \delta(x-f(t)) P-\delta(x-f(t)) m \frac{\mathrm{d}^{2} w(f(t), t)}{\mathrm{d} t^{2}}
\end{aligned}
$$

Here, $w(x, t)$ is the vertical deflection of the midline, $m$ is the moving mass, $N$ is the tension of the string, $\rho A$ is the mass density per unit length, $P$ is the external point force, and there is usually a gravitation force $m g$. The position of the moving point can be described with a quadratic function with respect to time

$$
f(t)=x_{0}+v t+\frac{1}{2} \dot{v} t^{2}
$$

where $x_{0}$ is an initial position of the mass. $v$ and $\dot{v}$ denote the mass velocity and acceleration respectively.

We impose initial conditions $w(x, 0)=0$,

$\partial w(x, t) /\left.\partial t\right|_{t=0}=0$ and boundary conditions $w(0, t)=0$, $w(l, t)=0$.

The Bernoulli-Euler beam is described by the equation

$$
\begin{aligned}
& E I \frac{\partial^{4} w(x, t)}{\partial x^{4}}+\rho A \frac{\partial^{2} w(x, t)}{\partial t^{2}} \\
= & \delta(x-f(t)) P-\delta(x-f(t)) m \frac{\mathrm{d}^{2} w(f(t), t)}{\mathrm{d} t^{2}}
\end{aligned}
$$

with initial conditions $w(x, 0)=0, \partial w(x, t) /\left.\partial t\right|_{t=0}=0$ and boundary conditions $w(0, t)=0, w(l, t)=0$, $\partial^{2} w(0, t) / \partial x^{2}=0, \partial^{2} w(l, t) / \partial x^{2}=0$, and the Timoshenko beam

$$
\begin{aligned}
& \rho A \frac{\partial^{2} w(x, t)}{\partial t^{2}}-\frac{G A}{k}\left(\frac{\partial^{2} w(x, t)}{\partial x^{2}}-\frac{\partial \psi(x, t)}{\partial x}\right) \\
= & \delta(x-f(t)) P-\delta(x-f(t)) m \frac{\mathrm{d}^{2} w(f(t), t)}{\mathrm{d} t^{2}} \\
& \rho I \frac{\partial^{2} \psi(x, t)}{\partial t^{2}}-E I \frac{\partial^{2} \psi(x, t)}{\partial x^{2}}-\frac{G A}{k}\left(\frac{\partial w(x, t)}{\partial x}-\psi(x, t)\right) \\
= & 0
\end{aligned}
$$

with the same boundary and initial conditions as for the Bernoulli-Euler beam. Here, $E I$ is the bending stiffness, $G A / k$ is the shear stiffness, $\rho I$ is the rotatory inertia of the cross section of the beam, and $\psi$ is the angle of 
rotation of the cross section.

In each type of problem we have identical inertial terms $\delta(x-f(t)) m \mathrm{~d}^{2} w(f(t), t) / \mathrm{d} t^{2}$. Below we will consider only this term, since the remaining parts of the equations are treated in the classical way by the finite element method.

Let us follow the direct derivation commonly carried out in the literature. The acceleration of a mass particle moving at a varying speed $v$ in the space-time domain is described by the Renaudot formula

$$
\begin{aligned}
\frac{\mathrm{d}^{2} w(f(t), t)}{\mathrm{d} t^{2}}= & \left.\frac{\partial^{2} w(x, t)}{\partial t^{2}}\right|_{x=f(t)}+\left.2 v \frac{\partial^{2} w(x, t)}{\partial x \partial t}\right|_{x=f(t)} \\
& +\left.v^{2} \frac{\partial^{2} w(x, t)}{\partial x^{2}}\right|_{x=f(t)}+\left.\dot{v} \frac{\partial w(x, t)}{\partial x}\right|_{x=f(t)}
\end{aligned}
$$

where $f(t)$ describes the position of the load. The above formula represents simply the chain rule of derivation. The corresponding parts of the equation describe the lateral acceleration, Coriolis acceleration, centrifugal acceleration and acceleration associated with the change of particle velocity. These names are generally not adequate in the case of all structures. Let us compare two different problems: the vibrations of a string and longitudinal vibrations of a bar. In both cases we have the identical governing equation. However, in the case of longitudinal displacements we can not call the forces described by terms of the equation as centrifugal or Coriolis.

\section{The Finite Element Carrying the Moving Mass Particle}

We must underline here that the derived matrices contribute only the point mass effects. They must be simply added to classical matrices elaborated for a structure, i.e. for a string or a beam. The full discrete motion equation is

$$
\begin{aligned}
& \left(M+M_{m}\right) \ddot{w}^{i+1}+\left(C+C_{m}\right) \dot{w}^{i+1}+\left(K+K_{m}\right) w^{i+1} \\
= & F^{i+1}+e_{m}^{i}
\end{aligned}
$$

Here, $M$ is the inertia matrix of a structure, $M_{m}$ is a moving mass matrix, added only to the inertia matrix of the element on which it travels. The same occurs in the case of a damping matrix of the structure $C$ and the point mass $C_{m}$, and in the case of a stiffness matrix of the structure $K$ and the point mass $K_{m}$. The vector $F^{i+1}$ is the vector of external forces established in time $t_{i+1}$ and $e_{m}^{i}$ is the right hand side vector resulting from the the mass inertia term, established at the beginning of the time interval $\left[t_{i}, t_{i+1}\right]$. We will concentrate our attention on the mass influence only, thus we will derive matrices $M_{m}, C_{m}, K_{m}$, and $e_{m}^{i}$ in the equation

$$
M_{m} \ddot{w}^{i+1}+C_{m} \dot{w}^{i+1}+K_{m} w^{i+1}=e_{m}^{i}
$$

The matrices of the finite element that carry the iner- tial particle are composed from two sets: matrices describing the element of a structure and matrices that incorporate the mass influence. Since the elemental matrices are commonly known, below we will consider only the influence of the moving mass.

The solution of this problem concerns a mass particle moving on a general finite element. This can be applied to all types of structures: strings, beams, plates, shells, etc. Below we will derive the resulting matrices which will then be applied and tested with an Euler and a Timoshenko beam.

Let us consider a finite element of length $b$ of the edge of the mass trajectory. The mass particle $m$ passes through the finite element with a varying velocity $v$ in the time interval $h$, starting at the point $x=x_{0}$ (Figure 2).

The equation of virtual energy which describes the motion of the inertial particle can be written in the following form

$$
\Pi_{m}=\int_{0}^{b} w^{*}(x) \delta(x-f(t)) m \frac{\mathrm{d}^{2} w(f(t), t)}{\mathrm{d} t^{2}} \mathrm{~d} x
$$

where a virtual displacements $w^{*}(x)$ can be described with a function

$$
w^{*}(x)=\left(1-\frac{x}{b}\right) w_{1}+\frac{x}{b} w_{2}
$$

Quantities marked by $(.)^{*}$ refer to a virtual state. $w_{1}$ and $w_{2}$ are nodal transverse virtual displacements at the ends of a finite element. We take first-order polynomials as the shape functions describing the interpolation of the displacements:

$$
w(x, t)=\left(1-\frac{x}{b}\right) w_{1}(t)+\frac{x}{b} w_{2}(t)
$$

Here, $w_{1}(t)$ and $w_{2}(t)$ are the nodal displacements in time. This is a natural assumption since the finite element edge is straight in the case of simple shape functions describing linear displacement distributions in the element. In such a case the third term of (5) reduces to zero. That is why we must write the Renaudot formula (5) in a different form:

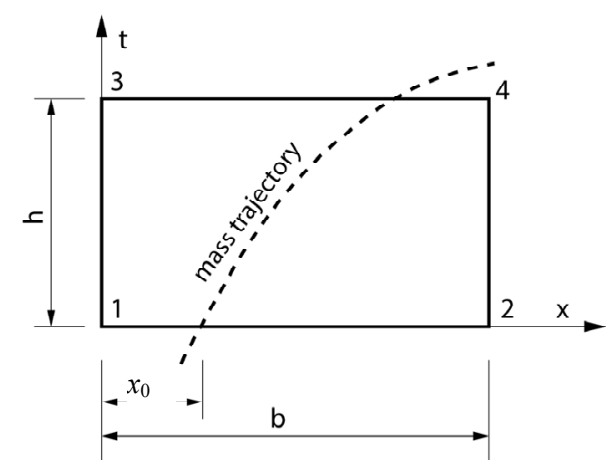

Figure 2. The mass trajectory in the space-time finite element. 


$$
\begin{aligned}
& \frac{\mathrm{d}^{2} w(f(t), t)}{\mathrm{d} t^{2}} \\
= & \left.\frac{\partial^{2} w(x, t)}{\partial t^{2}}\right|_{x=f(t)}+\left.v \frac{\partial^{2} w(x, t)}{\partial x \partial t}\right|_{x=f(t)} \\
& +\left.\dot{v} \frac{\partial w(x, t)}{\partial x}\right|_{x=f(t)}+v \frac{\mathrm{d}}{\mathrm{d} t}\left[\left.\frac{\partial w(x, t)}{\partial x}\right|_{x=f(t)}\right]
\end{aligned}
$$

The fourth term of (11) is developed in a Taylor series in terms of the time increment $\Delta t=h$

$$
\begin{aligned}
& {\left[\left.\frac{\partial w(x, t)}{\partial x}\right|_{x=f(t)}\right]^{t+h} } \\
= & {\left[\left.\frac{\partial w(x, t)}{\partial x}\right|_{x=f(t)}\right]^{t}+\left\{\frac{\mathrm{d}}{\mathrm{d} t}\left[\left.\frac{\partial w(x, t)}{\partial x}\right|_{x=f(t)}\right]\right\}^{t}(1-\alpha) h } \\
& +\left\{\frac{\mathrm{d}}{\mathrm{d} t}\left[\left.\frac{\partial w(x, t)}{\partial x}\right|_{x=f(t)}\right]\right\}^{t+h} \alpha h
\end{aligned}
$$

Upper indices indicate the time in which the respective terms are defined. We assume the backward difference formula ( $\alpha=1)$. In this case we have

$$
\begin{aligned}
& \left\{\frac{\mathrm{d}}{\mathrm{d} t}\left[\left.\frac{\partial w(x, t)}{\partial x}\right|_{x=f(t)}\right]\right\}^{t+h} \\
= & \frac{1}{h}\left[\left.\frac{\partial w(x, t)}{\partial x}\right|_{x=f(t)}\right]^{t+h}-\frac{1}{h}\left[\left.\frac{\partial w(x, t)}{\partial x}\right|_{x=f(t)}\right]^{t}
\end{aligned}
$$

According to Equations (2), (10) and (13), Equation (11) is given by the difference formula

$$
\begin{aligned}
& \frac{\mathrm{d}^{2} w}{\mathrm{~d} t^{2}} \\
= & \left(1-\frac{f(h)}{b}\right) \ddot{w}_{1}^{i+1}+\frac{f(h)}{b} \ddot{w}_{2}^{i+1}-\frac{v}{b} \dot{w}_{1}^{i+1}+\frac{v}{b} \dot{w}_{2}^{i+1}-\frac{\dot{v}}{b} w_{1}^{i+1}(1) \\
& +\frac{\dot{v}}{b} w_{2}^{i+1}-\frac{v}{b h} w_{1}^{i+1}+\frac{v}{b h} w_{2}^{i+1}+\frac{v}{b h} w_{1}^{i}-\frac{v}{b h} w_{2}^{i}
\end{aligned}
$$

The upper index denotes time layer. The energy (8), with respect to (9) and (14) can be written in quadratic form, which, after a classical minimization, results in the matrix Equation (7), where

$$
\begin{gathered}
M_{m}=m\left[\begin{array}{cc}
(1-\kappa)^{2} & \kappa(1-\kappa) \\
\kappa(1-\kappa) & \kappa^{2}
\end{array}\right] \\
C_{m}=\frac{m v}{b}\left[\begin{array}{cc}
-(1-\kappa) & 1-\kappa \\
-\kappa & \kappa
\end{array}\right]
\end{gathered}
$$

$$
K_{m}=\frac{m}{b}\left(\frac{v}{h}+\dot{v}\right)\left[\begin{array}{cc}
-(1-\kappa) & 1-\kappa \\
-\kappa & \kappa
\end{array}\right]
$$

and

$$
e_{m}=\frac{m v}{b h}\left[\begin{array}{c}
(1-\kappa)\left(w_{2}-w_{1}\right) \\
\kappa\left(w_{2}-w_{1}\right)
\end{array}\right]
$$

with coefficient $\kappa=\left(x_{0}+v h+\frac{1}{2} \dot{v} h^{2}\right) / b, 0<\kappa \leq 1$. Here, $\kappa$ is a parameter which defines the position of the mass in the element at the beginning of the time increment.

This determines the position of the mass at time $t=h$, related to the finite element length $b$. The different terms describe the transverse inertia force related to the vertical acceleration, the Coriolis force, and the centrifugal force. The matrix factors $M_{m}, C_{m}$, and $K_{m}$ can be called the mass, damping, and stiffness matrices. The last term $e_{m}$ describes the nodal forces at the beginning of the time interval $\left[t_{i}, t_{i}+\Delta t\right]$. We must emphasize here that the matrices (15)-(17) and the vector (18) contribute only the moving inertial particle effect. The matrices of the mass influence in a finite element of a structure must be added to the global system of equations. We notice that the matrices (15)-(17) differ from the matrices that cause the divergence of the solution in the case of direct differentiation of $(5)^{1}$.

\section{Numerical Results}

The scheme of computations is given in Figure 3:

The finite element is subjected at a mid-point to the force and the inertia parameter, i.e. the concentrated mass. The force vector, usually placed at the right hand side of the resulting system of algebraic equations, is simply distributed over neighbouring nodes (bending moments in the case of a beam must be considered in nodes as

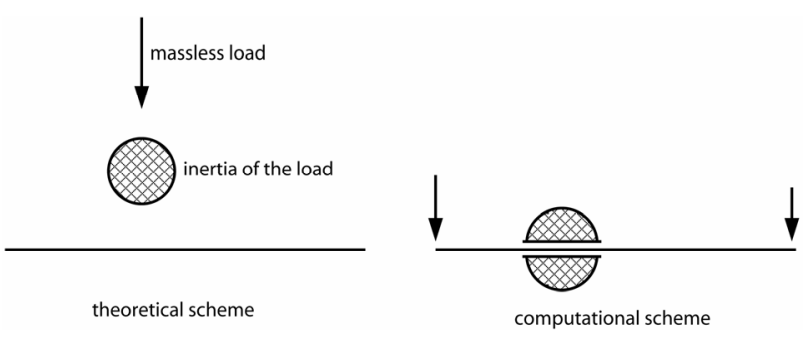

Figure 3. Theoretical scheme of the problem and the scheme assumed for computations.

$$
\begin{aligned}
& { }^{1} \text { The matrices that result in the divergence: } \\
& \qquad M_{m}=m\left[\begin{array}{cc}
(1-\kappa)^{2} & \kappa(1-\kappa) \\
\kappa(1-\kappa) & \kappa^{2}
\end{array}\right] \quad C_{m}=\frac{2 m v}{b}\left[\begin{array}{cc}
-(1-\kappa) & 1-\kappa \\
-\kappa & \kappa
\end{array}\right] \\
& K_{m}=\frac{m \dot{v}}{b}\left[\begin{array}{cc}
-(1-\kappa) & 1-\kappa \\
-\kappa & \kappa
\end{array}\right] \quad \kappa=\frac{x_{0}+v h+\frac{1}{2} \dot{v} h^{2}}{b}, 0<\kappa \leq 1 .
\end{aligned}
$$


well). The concentrated mass is incorporated directly into the left-hand-side matrices. Their coefficients vary in each time step and this requires the solution a system of equations once per time step. No iterations are required, unless unilateral contact is assumed. There are two gains in such a solution: more accurate and faster computations.

There are a few publications in which the inertial load moving on structures are considered directly numerically. The Timoshenko beam was described by Lee [18]. We will compare our curves with those results. Therefore, the data in the example is as follows: the length $L=1 \mathrm{~m}$, Young modulus $E=207 \mathrm{GPa}$, shear modulus $G=77.6$ GPa, mass density $\rho=7700 \mathrm{~kg} / \mathrm{m}^{3}$. The velocity

$v=(a \pi / L) \sqrt{E I /(\rho A)}$ was determined by the parameter

$\alpha$. Another parameter $\beta$ determines the cross section area $A=\beta^{2} L^{2} / \pi$ and cross section inertia moment

$I=\beta^{4} L^{4} /\left(4 \pi^{3}\right)$. The moving mass $m$ had values of $0.441 \mathrm{~kg}$ and $11.03 \mathrm{~kg}$. Trajectories of the moving mass point normalized to the static displacement of the simply supported Euler beam loaded in the middle by force $m g$ $\left(w_{s t}=m g L^{3} /(48 E I)\right)$ was presented in Figures 4-6. Figure 4 exhibits the dimensionless deflection of the simply supported Timoshenko beam under a moving mass for $\beta$ $=0.15$ and $\alpha=0.11,0.5$, and 1.1. This corresponds to the mass moving at $v=42.78,194.4$, and $427.7 \mathrm{~m} / \mathrm{s}$ on a relatively elastic beam. Lee solved the problem semi-analytically. A fourth order differential equation was solved by the Fourier transform and finally integrated by the Runge-Kutta method. In our test, we compare the results of Lee with our semi-analytically [19] obtained curves together with our Newmark time integration procedure applied to the finite element model of the Timoshenko beam. We notice a perfect coincidence of both solutions and quite good coincidence with Lee results.

Figure 5 shows the accuracy, which increases with the number of elements in the structure. Ten to twenty elements is sufficient in our example.

Another comparison was carried out between the Newmark and Houbolt methods. Both methods are sufficiently accurate. However, the curve for the Newmark method perfectly coincides with our semi-analytical results (Figure 6).

Now we will compare the displacements under a moving mass obtained with our approach with reference results by Stanisic and Sadiku [1,2]. The simply supported Bernoulli-Euler beam of length $L=6 \mathrm{~m}$, bending stiffness $E I /(\rho A)=275.4408 \mathrm{~m}^{4} / \mathrm{s}^{2}$, moving mass $m=$ $0.2 \rho A L$, velocity of $v=6 \mathrm{~m} / \mathrm{s}$ was assumed (Figure 7).

The simply supported Timoshenko beam was also considered in [4]. We compare our results with those published in the reference paper. Data were assumed as in [18], listed at the beginning of this section. The pa-

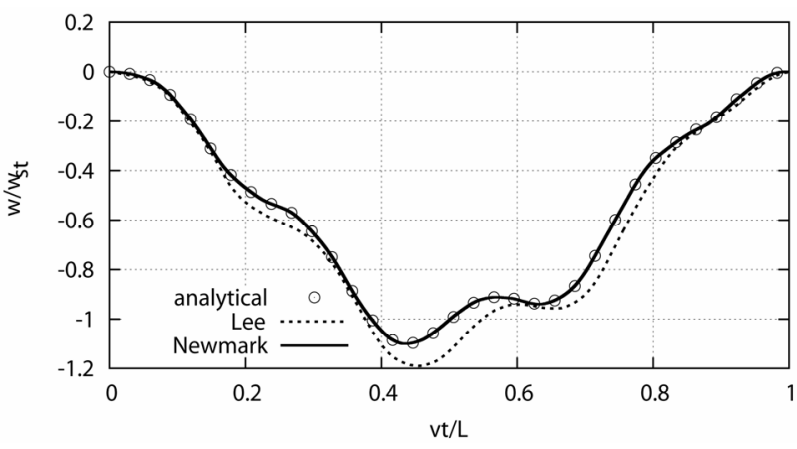

(a)

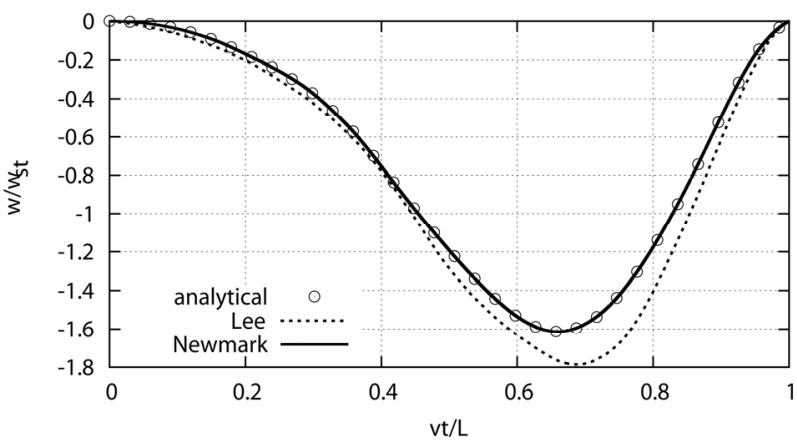

(b)

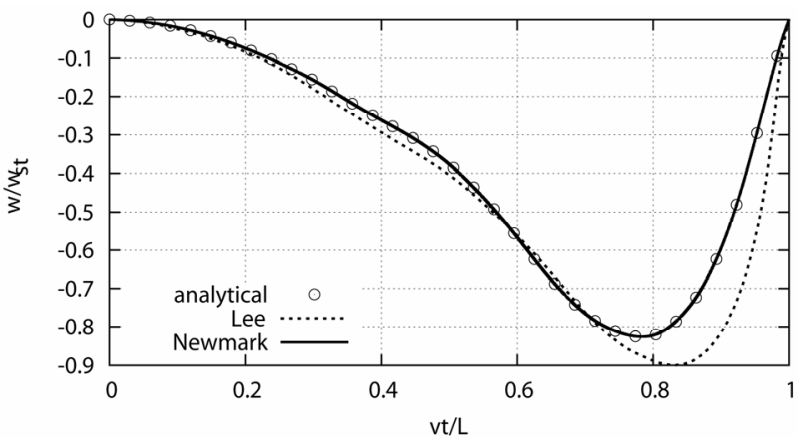

(c)

Figure 4. Normalized deflections of the simply supported Timoshenko beam under a moving mass particle for $\beta=$ 0.15: (a) $\alpha=0.11(v=42.78 \mathrm{~m} / \mathrm{s})$; (b) $\alpha=0.5(v=192.4 \mathrm{~m} / \mathrm{s})$; and (c) $\alpha=1.1(v=427.8 \mathrm{~m} / \mathrm{s})$.

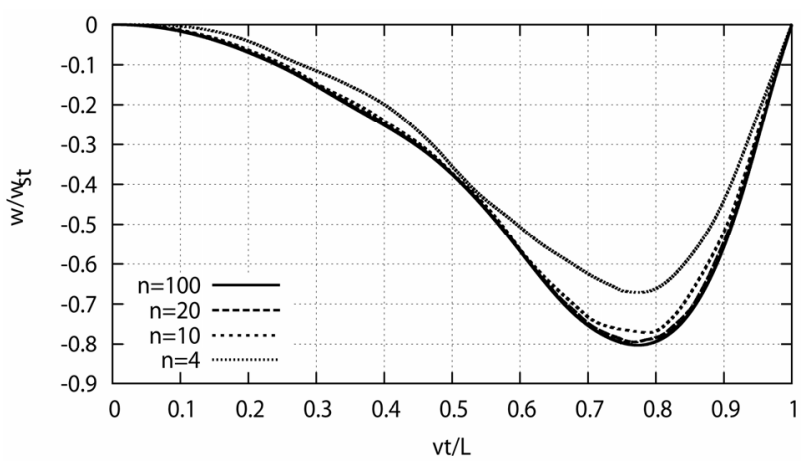

Figure 5. Accuracy of the Newmark method depending on the number of finite elements - displacements under a moving load $(\beta=0.03, \alpha=1.1)$. 


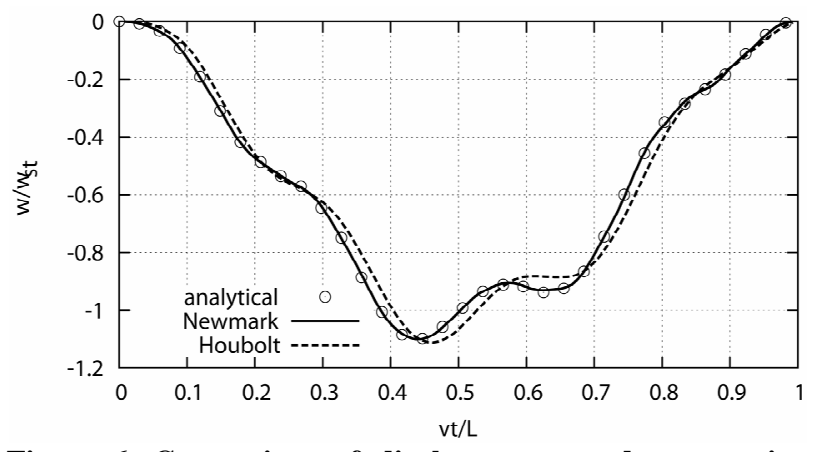

Figure 6. Comparison of displacements under a moving load computed with the Newmark and Houbolt method in the case of large time step $(\beta=0.15, \alpha=0.11)$.

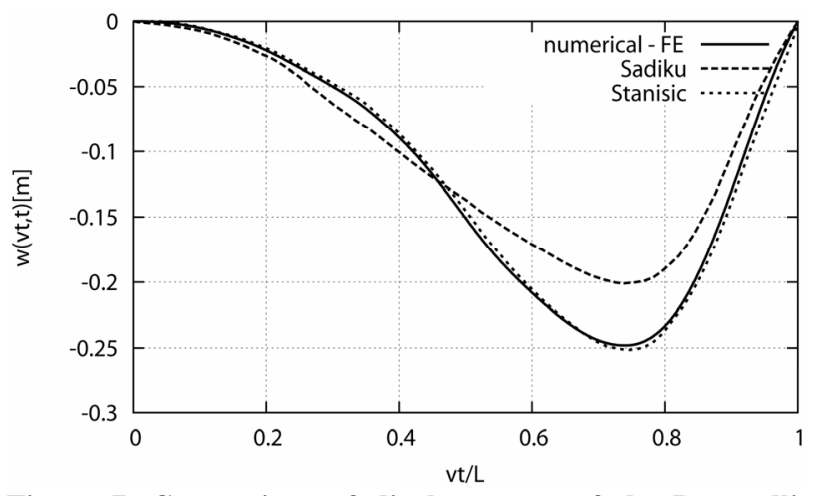

Figure 7. Comparison of displacements of the BernoulliEuler beam under the moving contact point with published by Stanisic [1] and Sadiku [2].

rameter $a=v / v_{c r}$, where the critical velocity $v_{c r}=(\pi / L) \sqrt{E I /(\rho A)}$. The acceleration $\dot{v}$ is is defined by the non-dimensional parameter $\kappa=\dot{v} \rho A L^{3} /(E I)$. Two cases were considered. First the case of $\beta=0.03$, $a=0.11$ was computed and depicted in Figure 8 for the acceleration $\kappa=1$, for a constant speed $\kappa=0$, and for a small retardation $(\kappa=-0.05)$. Figure 9 presents the case for a higher initial speed $a=0.5$ and $\beta=0.03$, for a constant speed $\kappa=0$, and with acceleration $\kappa=1$.

\section{Conclusion}

In this paper we proposed a new approach to the vibration analysis of structures subjected to a moving inertial particle by using the finite element method in space and a time integration method, for example the Newmark method, in time, here represented by the Newmark and Houbolt methods. Elements describing a moving mass particle (15)-(18) can be commonly used both in the analysis of the Euler beam and the Timoshenko beam. In engineering practice, most dynamic simulations are performed by the Newmark method. Each approach extends a group of problems that can be directly solved by this commonly used time integration method, and is valuable. We showed in the paper that these matrices result in ac-

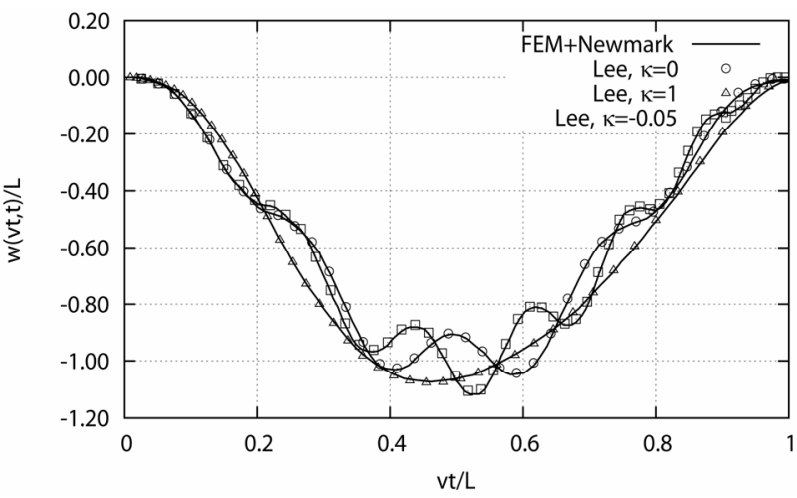

Figure 8. Comparison of displacements of the Timoshenko beam under a moving contact point with those published by Lee [4]-parameters $\beta=0.03, \alpha=0.11$.

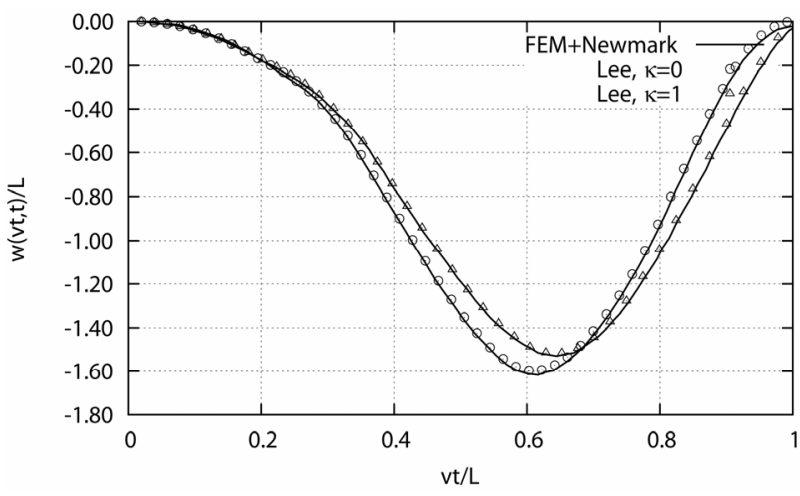

Figure 9. Comparison of displacements of the Timoshenko beam under a moving contact point with those published by Lee [4]-parameters $\beta=0.03, \alpha=0.5$.

curate and stable solutions of problems with a mass moving on a structure. Timoshenko beams or other shear resistant structures exhibit discontinuities in the solutions of the differential equations [19-21]. Although in practice nonlinear effects smooth the trajectories, high jumps of some physical quantities are observed. We assumed that identical computational results should be obtained both by analytical and numerical tools. There is no reason to say that numerical solutions converge to inaccurate results. Our finite element approach proves that simple elemental matrices derived from a mathematically correct analysis gives a perfect convergence to the analytical forms.

\section{REFERENCES}

[1] M. M. Stanisic, J. A. Euler and S. T. Montgomery, "On a Theory Concerning the Dynamical Behavior of Structures Carrying Moving Masses," Archive of Applied Mechanics, Vol. 43, No. 5, 1974, pp. 295-305. doi:10.1007/BF00537218

[2] S. Sadiku and H. H. E. Leipholz, "On the Dynamics of Elastic Systems with Moving Concentrated Masses," Ar- 
chive of Applied Mechanics, Vol. 57, No. 3, 1987, pp. 223-242.

[3] H. P. Lee, "On the Dynamic Behaviour of a Beam with an Accelerating Mass," Archive of Applied Mechanics, Vol. 65, No. 8, 1995, pp. 564-571. doi:10.1007/BF00789097

[4] H. P. Lee, "Transverse Vibration of a Timoshenko Beam Acted on by an Accelerating Mass," Applied Acoustics, Vol. 47, No. 4, 1996, pp. 319-330. doi:10.1016/0003-682X(95)00067-J

[5] V. A. Krysko, J. Awrejcewicz, A. N. Kutsemako and K. Broughan, "Interaction between Flexible Shells (Plates) and a Moving Lumped Body," Communications in Nonlinear Science and Numerical Simulation, Vol. 11, No. 1, 2006, pp. 13-43. doi:10.1016/j.cnsns.2004.06.006

[6] J. J. Wu, A. R. Whittaker and M. P. Cartmell, "The Use of Finite Element Techniques for Calculating the Dynamic Response of Structures to Moving Loads," Computers and Structures, Vol. 78, No. 6, 2000, pp. 789-799. doi:10.1016/S0045-7949(00)00055-9

[7] P. Lou, G. Dai and Q. Zeng, "Dynamic Analysis of a Timoshenko Beam Subjected to Moving Concentrated Forces Using the Finite Element Method," Shock and Vibration, Vol. 14, No. 6, 2007, pp. 459-468.

[8] J. Hino, T. Yoshimura, K. Konishi and N. Ananthanarayana, "A Finite Element Prediction of the Vibration of a Bridge Subjected to a Moving Vehicle Load," Journal of Sound and Vibration, Vol. 96, No. 1, 1984, pp. 45-53. doi:10.1016/0022-460X(84)90593-5

[9] Y. H. Lin and M. W. Tretheway, "Finite Element Analysis of Elastic Beams Subjected to Moving Dynamic Loads," Journal of Sound and Vibration, Vol. 136, No. 2, 1990, pp. 323-342. doi:10.1016/0022-460X(90)90860-3

[10] Y. S. Cheng, F. T. K. Au and Y. K. Cheung, "Vibration of Railway Bridges under a Moving Train by Using Bridge-Track-Vehicle Element," Engineering Structures, Vol. 23, No. 12, 2001, pp. 1597-1606. doi:10.1016/S0141-0296(01)00058-X

[11] A. V. Pesterev, L. A. Bergman, C. A. Tan, T.-C. Tsao and B. Yang, "On Asymptotics of the Solution of the Moving Oscillator Problem," Journal of Sound and Vibration, Vol. 260, No. 3, 2003, pp. 519-536. doi:10.1016/S0022-460X(02)00953-7
[12] D. M. Yoshida and W. Weaver, "Finite-Element Analysis of Beams and Plates with Moving Loads," Journal of the International Association Bridge and Structural Engineering, Vol. 31, No. 1, 1971, pp. 179-195.

[13] F. V. Filho, "Finite Element Analysis of Structures under Moving Loads," The Shock and Vibration Digest, Vol. 10, No. 8, 1978, pp. 27-35. doi: $10.1177 / 058310247801000803$

[14] A. O. Cifuentes, "Dynamic Response of a Beam Excited by a Moving Mass," Finite Elements in Analysis and Design, Vol. 5, No. 3, 1989, pp. 237-246. doi:10.1016/0168-874X(89)90046-2

[15] J. R. Rieker, Y. H. Lin and M. W. Trethewey, "Discretization Considerations in Moving Load Finite Element Beam Models," Finite Elements in Analysis and Design, Vol. 21, No. 3, 1996, pp. 129-144. doi:10.1016/0168-874X(95)00029-S

[16] C. I. Bajer and B. Dyniewicz, "Space-Time Approach to Numerical Analysis of a String with a Moving Mass," International Journal for Numerical Methods in Engineering, Vol. 76, No. 10, 2008, pp. 1528-1543.

[17] C. I. Bajer and B. Dyniewicz, "Virtual Functions of the Space-Time Finite Element Method in Moving Mass Problems," Computers and Structures, Vol. 87, No. 7-8, 2009, pp. 444-455. doi:10.1016/j.compstruc.2009.01.007

[18] H. P. Lee, "The Dynamic Response of a Timoshenko Beam Subjected to a Moving Mass," Journal of Sound Vibration, Vol. 198, No. 2, 1996, pp. 249-256. doi:10.1006/jsvi.1996.0567

[19] B. Dyniewicz and C. I. Bajer, "New Feature of the Solution of a Timoshenko Beam Carrying the Moving Mass Particle," Archive of Mechanics, Vol. 62, No. 5, 2010, pp. 327-341.

[20] B. Dyniewicz and C. I. Bajer, "Paradox of the Particle's Trajectory Moving on a String," Archive Applied Mechanics, Vol. 79, No. 3, 2009, pp. 213-223. doi:10.1007/s00419-008-0222-9

[21] B. Dyniewicz and C. I. Bajer, "Discontinuous Trajectory of the Mass Particle Moving on a String or a Beam," Machine Dynamics Problems, Vol. 31, No. 2, 2007, pp. 66-79. 